shown previously to be biocompatible and nonadhesive to protein and cells. Details of the rubber casting and UV embossing techniques were previously published.

The researchers found that the TiN mold can be used for more than 20 rubber castings without much dimensional change, which they attribute to the low surface energies of both TiN and silicone. Scanning probe microscopy (SPM) analysis shows that the roughness of the TiN master is about $4 \mathrm{~nm}$, which the researchers present as evidence that FIB lithography of TiN produces surfaces smooth enough for nanopatterning. Similarly, successive generations of rubber moldings display little dimensional difference. However, scanning electron micrographs show that the structure resulting from the eighth consecutive PEGDA embossing (from the same silicone mold) displays some surface defects, although the major dimensions are replicated well. The researchers believe that increasing the hydrophobicity of the silicone and/or PEGDA can minimize surface defects.

The researchers said that their technique is inexpensive and requires no special processing conditions, thereby allowing several tens to hundreds of UV-embossed PEGDA replications to be easily made from a single submicrometer-patterned TiN master mold.

STEVEN TROHALAKI

\section{Theoretical Simulation of Laser Emissions Describes Intensity and Polarization Properties of Semiconductor Nanowires}

Nanowires, which can have diameters of 20-200 nm, are capable of laser emission through the axial direction. While the smallerdiameter nanowires can only support emission with a mode similar to the fundamental hybrid mode of optical fibers, larger-diameter nanowires can give rise to different coexisting modes. Information on the properties of the far-field radiation is necessary to reveal the particular mode type involved. As a first approach toward analyzing the emissive modes of nanowire lasers, A.V. Maslov and C.Z. Ning from the Center for Nanotechnology at NASA Ames Research Center in California have developed a theoretical methodology to establish the mode type, deduced from the characteristics of the corresponding far-field radiation. They presented their research in the March 15 issue of Optics Letters.

Maslov and Ning applied the finite-difference time-domain method to formulate their simulation. Initially, a current source excited two wave packets that traveled along the length of a vertically oriented nanowire toward the two ends of the nanowire, respectively. In their discussion, the researchers assumed that in a self-supporting nanowire in a steady-state regime, it was adequate to consider only the radiation that is incident on the top end. They then defined a domain using cylindrical coordinates: 100 cells in the $\rho$ direction, 600 cells in the $z$ direction, and a radius of 10 cells. After an excited wave packet arrived at the top of the nanowire, part of it was reflected, and the remainder left the nanowire so that an electronic field with two components, $E_{\theta}$ and $E_{\phi}$, was created in the farfield region, where $\theta$ is the polar or elevation angle with respect to the $z$ axis and $\varphi$ is the azimuth angle. These were calculated by integrating an expression for the equivalent current on the surface that surrounds the source of the fields. The value of the dielectric constant was assumed as 6, typical of nanowire materials such as $\mathrm{GaN}, \mathrm{ZnO}$, and $\mathrm{CdS}$ at emitting frequencies. The researchers then analyzed the spectral density of the emitted radiation. Analysis of the resulting expressions was related to the polarization properties by considering the symmetry of the calculated far-field patterns. For example, the transverse magnetic-field polarization $\left(T M_{01}\right)$ mode had only an $E_{\theta}$ component, the transverse electricfield polarization $\left(T E_{01}\right)$ mode had only an $E_{\phi}$ component, and the $H E_{11}$ mode (i.e., the fundamental hybrid mode) had both $E_{\theta}$ and $E_{\phi}$ components.

\section{Know the \\ Ups and Downs ...}

Steep slope

+ Big height difference

+ Large scan field

The LSM 5 PASCAL confocal laser scanning microscope broadens your microscopic horizons.
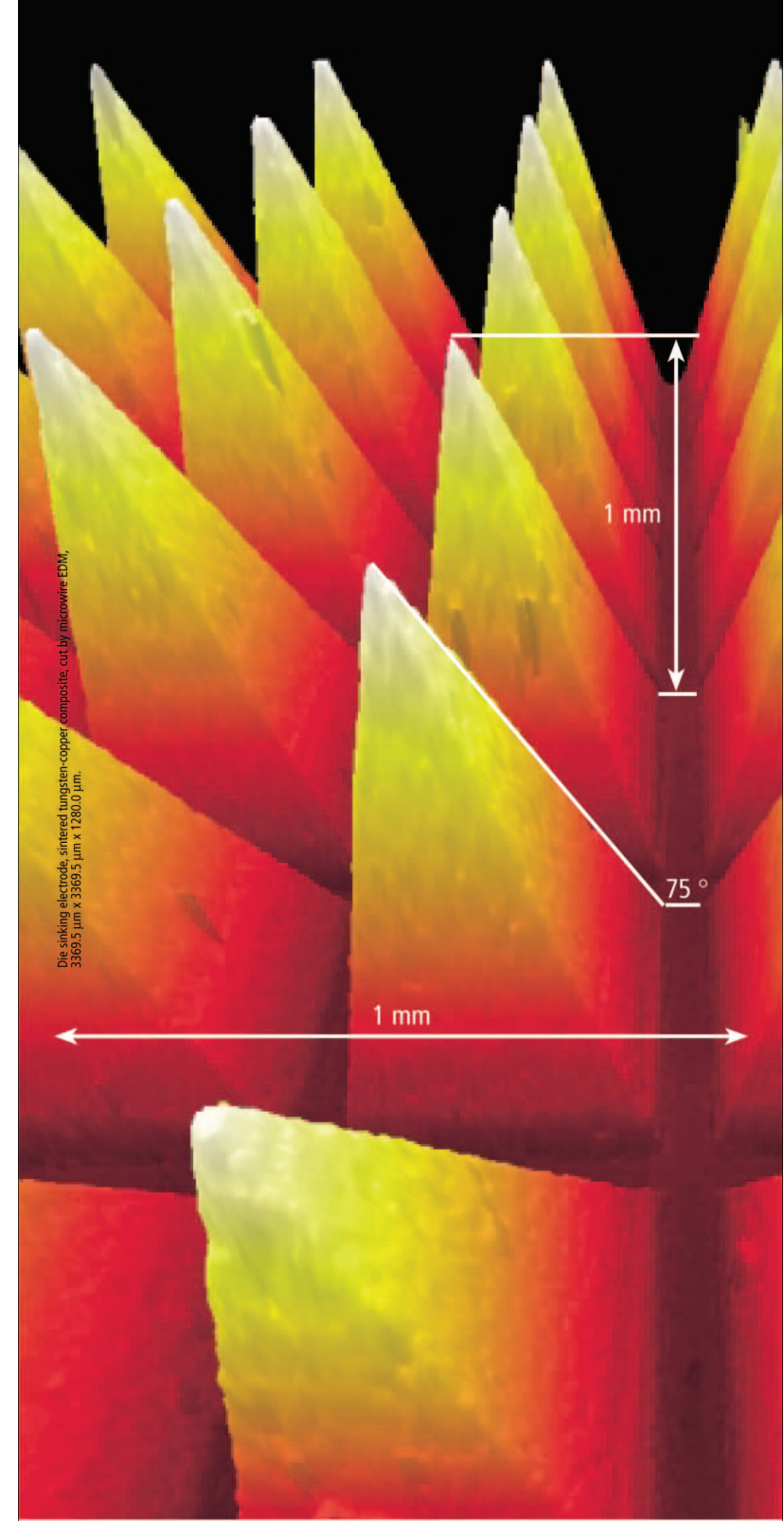

Highly resolved images Simple and efficient. Non-destructive.

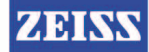

www.zeiss.com/lsm-mat

We make it visible. 
Evaluating the intensity for the different modes gave a minimum of zero emission at $\theta=0$ for the $T E_{01}$ and $T M_{01}$ modes, and maxima in the range of $30-50^{\circ}$. On the other hand, the $H E_{11}$ mode had a maximum emission at $\theta=0$. The radius of the nanowire directly affected the intensity of the response of each mode, but much more for the $T M_{01}$ and $H E_{11}$ modes than for the $T E_{01}$ mode. Experimentally, polarization measurements will determine the mode type and should be taken close to the top end of the nanowire to avoid interference with the radiation from the bottom end.

The researchers said that the ability to control the properties of laser emission from nanowires may open new possibilities in optoelectronics in conjunction with self-waveguiding and the possibility of nanowire array fabrication.

SIARI SOSA

\section{Simulation of Spin-MOSFET Predicts Strong Dependence of Spin Transport on Magnetic Configuration}

Spin transistors utilizing two ferromagnetic layers as spin injector and spin analyzer, respectively, possess unique output characteristics that are controlled by the relative magnetization configuration of the ferromagnets as well as bias conditions. Together with the ability to utilize the latter as nonvolatile binary data, spin transistors could allow the fabrication of ultrahigh-density nonvolatile memory in integrated circuits (ICs) in which the memory cell consists of a single spin transistor as well as functional spin-transistorgate-based nonvolatile reconfigurable logic. In order to attain high-performance "spintronic" integrated circuits, five requirements need to be fulfilled: (1) a large magnetocurrent ratio for nonvolatile memory and logic functions; (2) high transconductance for high-speed operation; (3) a high amplification capability (voltage, current, and/or power gains) to restore propagating signals between transistors; (4) a small power-delay product and small off current for low power dissipation; and (5) a simple device structure for a high degree of integration and high process yield.

Until now, proposed spin transistor designs have been unable to satisfy all of these requirements. The simultaneous achievement of high transconductance and amplification capability with a large magnetocurrent ratio has been difficult. S. Sugahara and M. Tanaka of the Department of Electrical Engineering at the University of Tokyo, however, have conducted a two-dimensional numerical analysis of their spin-type metal oxide semiconductor field-effect transistor (MOSFET) design, fulfilling all five of these requirements and predicting highamplification capability, a low powerdelay product, a low off current, and extremely large magnetocurrent ratios.

As reported in the March 22 issue of Applied Physics Letters, the designed spin MOSFET consists of a MOS gate structure and half-metallic ferromagnet (HMF) contacts for the source and drain, similar to a Schottky source/drain MOSFET except for the HMF source/drain contacts, which are $\mathrm{HMF} / \mathrm{Si}$ junctions without $p-n$ junctions. The device simulated used a channel length of $30 \mathrm{~nm}$, a Si layer thickness of $10 \mathrm{~nm}$, and an oxide layer thickness of 2-3 $\mathrm{nm}$. The design can be applied not only to $n$ - and $p$-channel devices, but also to accumulation- and inversion-type channel devices. Promising HMF materials include Heusler alloys and oxides such as $\mathrm{CrO}_{2}$ and $\mathrm{Fe}_{2} \mathrm{O}_{3}$. Of even more interest are halfmetallic compounds exhibiting the zincblende crystal structure, such as CrAs, $\mathrm{MnAs}$, and $\mathrm{CrSb}$, since their predicted high Curie temperature and large bandgap of the insulating spin band are most useful as HMF source/drain materials, said the researchers.

Sugahara and Tanaka found that their simulations predict that the HMF source not only functions as a contact for blocking an off current, but also acts as a spin injector of up-spin electrons from the HMF source to the channel. The HMF drain selectively extracts the up-spin electrons from the channel when the magnetizations of the HMF source and drain are parallel. The scientists expect an extremely large magnetocurrent ratio due to the high spinselectivity of the HMF source/drain when these spin-filter effects are combined. The output characteristics were calculated using the Tsu-Esaki formula with a twodimensional transmission probability calculation and gave magnetocurrent ratios of $>1000 \%$ at $<1.0 \mathrm{~V}$ drain/source bias $V_{\mathrm{DS}}$ and even larger ratios at smaller $V_{\mathrm{DS}}$. Other calculated device parameters such as transconductance were similar to those that have been obtained for MOSFETS with gate lengths of $<100 \mathrm{~nm}$.

Alfred A. ZiNN

\section{Effects of Chirality on Goos-Hänchen Phase Shifts at Interfaces in Optical Media Explored}

A light beam that is totally reflected at the interface between two different media always undergoes a phase change. Light waves with different incident wave vectors are subject to different magnitudes of this phase change. As a result of this inter- ference among the plane-wave components with different incident wave vectors, the weight center of the reflected beam is displaced laterally with respect to the weight center of the incident beam, a phenomenon known as the Goos-Hänchen shift. This event includes both a phase shift that can either reinforce or oppose the incident wave vector and a lateral beam shift that is either in the same or opposite direction as the horizontal component of the energy flux of the incident beam. Recently, D.-K. Qing and G. Chen from the Department of Mechanical Engineering at the Massachusetts Institute of Technology investigated Goos-Hänchen shifts at the interfaces between optical media of different "handedness," or chirality.

The Goos-Hänchen shifts are well known for total reflection of light propagating in conventional dielectric materials. Such media are known as "righthanded" materials, since the wave vector, electric-field vector, and magnetic vector of light beams traveling in them form a right-handed system. However, in media that have negative permeability and negative permittivity, these vectors form a lefthanded system, and thus the medium is called "left-handed." Although left-handed materials have attracted recent interest for new devices such as novel optical waveguides, superlenses, and microcavities, no previous study exists in which the light propagating through them was incident from the left-handed medium.

As reported in the April 15 issue of Optics Letters, Qing and Chen have determined mathematically the Goos-Hänchen phase changes and lateral beam shifts for beams propagating in both left-handed and right-handed media, reflected at interfaces between both same-handed and different-handed materials. This gives four possible situations. In a concise diagram, the researchers elucidate the differences between Goos-Hänchen shifts for each possible system. When the two materials are both of the same handedness (whether left- or right-handed), the Goos-Hänchen phase shift opposes the phase change resulting from propagation through the incident medium. This change is positive in right-handed systems and negative in left-handed ones. The Goos-Hänchen lateral shift between two same-handed systems is in the same direction as the horizontal component of the incident energy flux. Alternatively, when the two media are of opposite handedness, the Goos-Hänchen phase shift reinforces the phase variation of the incident beam, while the lateral shift is opposite the horizontal component of the incident energy flux. These observations are true for inter- 